\title{
Análise fitoquímica e atividade antimicobacteriana de extratos metanólicos de Jacaranda cuspidifolia Mart. (Bignoniaceae)
}

\begin{abstract}
ARRUDA, A.L.A. ${ }^{1 *}$; SOUZA, D.G. ${ }^{1}$; VIEIRA, C.J.B. ${ }^{1}$; OLIVEIRA, R.F. ${ }^{1}$; PAVAN, F.R. ${ }^{2}$; FUJIMURA, C.Q.L. ${ }^{2}$; RESENDE, U.M. ${ }^{3}$; CASTILHO, R.O. ${ }^{4}$

${ }^{1}$ Universidade Católica Dom Bosco (UCDB), Curso de Farmácia, Centro de Ciências Biológicas e da Saúde, CEP: 79002-173, Campo Grande-Brasil *analu_arruda@hotmail.com 22Universidade Estadual Paulista Júlio de Mesquita Filho, Faculdade de Ciências Farmacêuticas, Departamento de Ciências Biológicas, CEP: 14801-902, AraraquaraBrasil ${ }^{3}$ Universidade Federal de Mato Grosso do Sul (UFMS), Departamento de Biologia, Centro de Ciências Biológicas e da Saúde, Caixa Postal 549, CEP: 79070-900, Campo Grande-Brasil ${ }^{4}$ Universidade Federal de Minas Gerais, Faculdade de Farmácia, Departamento de Produtos Farmacêuticos, CEP: 31270-901, Belo Horizonte-Brasil
\end{abstract}

\begin{abstract}
RESUMO: Jacaranda cuspidifolia Mart., conhecida popularmente como "caroba", "jacarandá" ou "bolacheira", é utilizada medicinalmente para o tratamento da sífilis e da gonorréia. A atividade antimicobacteriana dessa espécie foi avaliada em ensaios in vitro com os extratos metanólicos das cascas e folhas, segundo o Método Analítico Alamar Blue (MABA). Os valores de concentração inibitória mínima para os extratos metanólicos das cascas e das folhas de J. cuspidifolia foram iguais a $\mathrm{CIM}=250 \mu \mathrm{g} \mathrm{mL}^{-1}$ para ambos os extratos. A análise fitoquímica, por Cromatografia em Camada Delgada de gel de sílica, dos extratos metanólicos das cascas e folhas revelou a presença de taninos, flavonóides, terpenos, cumarinas e esteróides. A análise dos perfis dos extratos metanólicos por Cromatografia Líquida de Alta Eficiência de Fase Reversa registrou a presença de compostos fenólicos derivados do verbascosídeo sugerindo a provável responsabilidade pela ação antimicobacteriana.
\end{abstract}

Palavras-chave: Jacaranda cuspidifolia Mart., Mycobacterium tuberculosis $\mathrm{H}_{37} \mathrm{RvA}$, atividade antimicobacteriana, compostos fenólicos, Verbascosídeo

\begin{abstract}
Phytochemical analysis and antimycobacterial activity of methanol extracts from Jacaranda cuspidifolia Mart. (Bignoniaceae). Jacaranda cuspidifolia Mart., popularly known as "caroba", "jacaranda" or "bolacheira", is used as medicine for the treatment of syphilis and gonorrhea. The antimycobacterial activity of this species was assessed by means of in vitro assays with methanol extracts of barks and leaves according to the Microplate Alamar Blue Assay (MABA). The minimal inhibitory concentration values for methanol extracts of barks and leaves from J. cuspidifolia were MIC $=250 \mu \mathrm{g} \mathrm{mL}^{-1}$ for both extracts. Phytochemical analysis, by Thin Layer Chromatography on silica gel, of methanol extracts of barks and leaves revealed the presence of tannins, flavonoids, terpenes, cumarins and steroids. Analysis of the profiles of methanol extracts by High Performance Liquid Chromatography - Reversed Phase recorded the presence of phenolic compounds derivatives of verbascoside, suggesting their probable responsibility for the antimycobacterial action.
\end{abstract}

Key words: Jacaranda cuspidifolia Mart., Mycobacterium tuberculosis $\mathrm{H}_{37} \mathrm{RvA}$, antimycobacterial activity, phenolic compounds, Verbascoside

\section{INTRODUÇÃO}

A tuberculose é considerada grave problema dentro da saúde pública, voltando a ocupar lugar de destaque entre as principais doenças infectocontagiosas. O aparecimento cada vez mais comum de bacilos resistentes ou multi-resistentes e o surgimento da Síndrome da Imunodeficiência Adquirida (SIDA) tem contribuído para o surgimento, cada vez maior, de novos casos da doença (Souza \& Vasconcelos, 2005). Dados da Organização Mundial da Saúde revelam aumento de 8,3 milhões para 9,24 milhões de casos novos da doença, no período de 2000 a 2006 (OMS, 2009).

A descoberta de novas drogas para o tratamento da tuberculose constitui grande desafio,

Recebido para publicação em 14/04/2010

Aceito para publicação em 24/02/2012

Rev. Bras. PI. Med., Botucatu, v.14, n.2, p.276-281, 2012. 
pois as micobactérias são consideradas organismos de crescimento lento, patogênicas e a parede, rica em lipídeos, representam verdadeira proteção contra os agentes agressores (Rando et al., 2002; Arantes et al., 2005).

Plantas constituem uma das principais fontes de metabólitos ativos com ação antimicobacteriana (Okunade et al., 2004; Mcgaw et al., 2008). Diversas substâncias já têm a atividade antimicobacteriana estabelecida, como é o caso dos terpenos (Cantrell et al., 2001; Januário et al., 2002; Arantes et al., 2005); alcalóides (Kanokmedhakul et al., 2002; Newton et al., 2002; Sunthitikainsakul et al., 2003; Gibbons et al., 2003); flavonóides (Fris-Moller et al., 2002; Murillo et al., 2003; Schinkovitz et al., 2003); esteróides e saponinas (Schi et al., 2002; Januário et al., 2002) e fenóis e polifenóis (Asres et al., 2001; Seephonaki et al., 2002).

A família Bignoniaceae é constituída por 120 gêneros e 800 espécies encontradas principalmente na América do Sul e na África. Espécies dessa família têm hábito constituído por árvores, arbustos, subarbustos, lianas e raramente ervas. Muitas destas árvores são conhecidas pelo uso industrial (Tabebuia, Paratecoma, Parmenteiera, Catalpa e Jacaranda) ou como plantas ornamentais (Jacaranda, Catalpa e Sphathodea) (Parker, 2009).

Jacaranda cuspidifolia Mart. pertence a família Bignoniaceae e tribo Tecomae, contém 49 espécies mundiais, sendo 39 destas espécies endêmicas no Brasil (Gachet \& Schüly, 2009). É conhecida popularmente como jacarandá, caroba e bolacheira e considerada árvore de médio porte com altura de 3-10 metros, apícola, utilizada na arborização e ornamentação de ruas, principalmente devido a beleza das flores arroxeadas. Produz anualmente grande quantidade de sementes viáveis, amplamente dispersas pelo vento, florescendo durante os meses de setembro/dezembro com as plantas totalmente despidas de folhagem velha. Apresenta propriedade inseticida, sendo a raiz usada no tratamento da sarna. É depurativa do sangue e usada em infecções bacterianas, como sífilis e blenorragia. A madeira, casca e folha são usadas no combate à febre (Pott \& Pott, 1994; Lorenzi, 2000; Scalon et al., 2006).

Dentre as espécies pertencentes ao gênero Jacaranda apenas 6 foram estudadas sob o ponto de vista fitoquímico. São elas a Jacaranda acutifolia, Jacaranda caucana, Jacaranda copaia, Jacaranda decurrens, Jacaranda mimosifolia e Jacaranda filicifolia e as principais classes de substâncias isoladas foram terpenóides, quinonas, flavonóides, ácidos graxos, cetosídeos e um dímero feniletanóide (Gachet \& Schüly, 2009).

O presente trabalho teve como objetivo obter o perfil fitoquímico dos extratos metanólicos das cascas e das folhas de Jacaranda cuspidifolia Mart. e avaliar a atividade antimicobacteriana in vitro desses extratos contra a cepa padrão Mycobacterium tuberculosis $\mathrm{H}_{37} \mathrm{Rv}$.

\section{MATERIAL E MÉTODO}

\section{Material vegetal}

A espécie Jacaranda cuspidifolia Mart. foi selecionada de acordo com critérios etnofarmacológicos e as cascas e as folhas foram coletadas em novembro de 2006 numa área de cerrado, em Aquidauana (MS). O material vegetal foi identificado pela botânica Dra Ubirazilda Maria Resende e uma amostra foi depositada no Herbário da Universidade Federal de Mato Grosso do Sul (HMS), sob o número 11923.

\section{Preparo dos extratos}

Após a coleta, as cascas e as folhas foram dessecadas em estufa de circulação forçada de ar durante três dias na temperatura de $40^{\circ} \mathrm{C}$ e depois trituradas em moinho tipo Willey. O material vegetal foi extraído com hexano e metanol p.a. por maceração a frio até o esgotamento e secos em evaporador rotatório. Os rendimentos dos extratos metanólicos das cascas (EMC) e das folhas (EMF) de Jacaranda cuspidifolia Mart. foram de $7,65 \%$ e $11,30 \%$, respectivamente.

\section{Análise fitoquímica dos extratos metanólicos das cascas e folhas}

A presença de terpenos, alcalóides, flavonóides, cumarinas e esteróides foram avaliados nos extratos metanólicos das cascas (EMC) e folhas (EMF), por Cromatografia em Camada Delgada com gel de sílica, de acordo com Wagner et al. (1984).

\section{Caracterização dos extratos metanólicos (EMC e EMF) por CLAE}

Para a cromatografia líquida de alta eficiência, utilizou-se cromatógrafo líquido de alta eficiência Waters ${ }^{\circledR}$, composto de injetor automático, modelo 2695, com sistema operacional computadorizado com software Empower e detector de arranjos de diodos (DAD), modelo 2996, bomba modelo L-6200a, integrador modelo C-R4A. Coluna para CLAE ODS C-18 LiChrospher $^{\circledR}(125 \times 4,0 \mathrm{~mm}, 5 \mu \mathrm{m})$, Merck e pré-coluna LiChrospher ${ }^{\circledR} 100 \mathrm{RP}-18(5 \mu \mathrm{m})$. As amostras foram pesadas em frascos de Eppendorf e solubilizadas em metanol grau CLAE, na concentração de $10 \mathrm{mg} \mathrm{mL}^{-1}$ para os extratos metanólicos da casca (EMC) e da folha (EMF) e 1 $\mathrm{mg} \mathrm{mL}^{-1}$ para as substâncias de referência. As amostras foram filtradas em millex LCR com membrana PTFE modificada para filtração de 
solventes orgânicos e aquosos 0,45 $\mu \mathrm{m}, 13 \mathrm{~mm}$. Alíquotas de $10 \mu \mathrm{L}$ das amostras foram injetadas de modo automático a temperatura de $40^{\circ} \mathrm{C}$ e velocidade de fluxo de $1 \mathrm{~mL} \mathrm{~min}{ }^{-1}$. A detecção foi realizada em detector de arranjo de diodos a $\lambda 210 \mathrm{~nm}$. Espectros de UV de $\lambda 210$ a $400 \mathrm{~nm}$ foram obtidos on line para identificação de cada pico. Gradiente linear de $\mathrm{H}_{2} \mathrm{O}$ (A) e $\mathrm{CH}_{3} \mathrm{CN}$ (B) foi empregado $0 \mathrm{~min} 95 \%$ de A e $5 \%$ B; 60 min $60 \%$ de A, $40 \%$ de $B$, seguido de 5 min de gradiente linear de $95 \%$ de $A$ e $5 \%$ de $B$. Os solventes utilizados foram grau CLAE (TÉDIA).

\section{Micobactéria empregada}

Cultura confluente crescida no meio de Lowestein-Jensen (LJ) da cepa padrão de Mycobacterium tuberculosis $\mathrm{H}_{37}$ RvATCC 27294 foi mantida sob refrigeração até o momento do uso. Para o ensaio foi retirada uma alçada que foi semeada no meio de Middlebrook 7H9 com incubação por 10 dias. A cultura foi diluída até a turvação correspondente a turvação do tubo 1 da escala de MacFarland e a partir desta, realizada a diluição de 1:25 que foi empregada como suspensão inoculante.

\section{Determinação da atividade antimico- bacteriana}

Para determinar a atividade antimicobacteriana a partir da solução estoque, os extratos metanólicos das cascas (EMC) e folhas (EMF) de Jacaranda cuspidifolia Mart. foram diluídos para obter concentrações de 4000 a $62,5 \mu \mathrm{g} \mathrm{mL}^{-1}$. A atividade foi determinada em triplicata utilizando microplacas estéreis de 96 poços (Falcon 3072; Recton Dickinson, Lincoln Park, NJ) e o método do Alamar Blue (MABA), segundo Franzblau et al. (1998). A Concentração Inibitória Mínima (CIM) foi definida como a menor concentração da droga capaz de prevenir a alteração de cor do reagente Alamar Blue (Acccumed International, Westlake, Ohio) de azul para rosa. A cor azul no poço foi interpretada como ausência de crescimento da micobactéria e a cor rosa como de viabilidade e multiplicação bacilar. Isoniazida $(0,03$ $\mu \mathrm{g} \mathrm{mL}^{-1}$ ) foi usada como controle.

\section{RESULTADO E DISCUSSÃO}

A avaliação da atividade antimicobacteriana para os extratos de J. cuspidifolia, mostrou Concentração Inibitória Mínima de $250 \mu \mathrm{g} \mathrm{mL}^{-1}$ para as cascas e para as folhas. Esse valor foi considerado superior ao da Isoniazida $\left(0,03 \mu \mathrm{g} \mathrm{mL}^{-1}\right)$ e ao da pirazinamida, um agente antimicobacteriano de primeira linha, que apresenta valor de CIM de $100 \mu \mathrm{g}$ $\mathrm{mL}^{-1}$ para o M. tuberculosis (Higuchi et al., 2008)

Fluorquinolonas tem apresentado alta eficácia no tratamento da tuberculose, principalmente, aquelas causadas por cepas de micobactérias multi- resistentes. Os valores de Concentração Inibitória Mínima para o ciprofloxacino e ofloxacina foi de 1,0 $\mathrm{mg} \mathrm{L}^{-1}$, enquanto que para o levofloxacino e moxifloxacino foram encontrados valores de CIM iguais a $0,5 \mathrm{mg} \mathrm{mL}^{-1}$ (Rodriguez et al., 2001; Díaz et al., 2003).

Muitas plantas são usadas pela população que habitam as áreas do cerrado para o tratamento de infecções causadas por microrganismos, incluindo a tuberculose (Almeida et al., 1998). Em estudos realizados por Pavan et al. (2009) foram avaliados os extratos clorofórmicos e metanólicos de 37 espécies de plantas distribuídas em 17 famílias do Cerrado Brasileiro. Os resultados obtidos demonstraram que os extratos brutos de dezenove plantas foram considerados ativos contra o Micobacterium tuberculosis $\mathrm{H}_{37} \mathrm{Rv}$.

Estudo da atividade antimicobacteriana realizados com extratos obtidos da espécie Jacaranda mimosaefolia contra Micobacterium phlei mostraram inibição (Mahran et al., 1991).

A prospecção fitoquímica dos extratos vegetais de Jacaranda cuspidifolia detectou a presença de taninos, flavonóides, terpenos, cumarinas e esteróides.

Estudos químicos dos constituintes do gênero Jacaranda foram descritos para seis espécies: Jacaranda acitufolia, Jacaranda caucana, Jacaranda copaia, Jacaranda decurrens, Jacaranda filicifolia e Jacaranda mimosifolia. As principais substâncias isoladas destas espécies foram triterpenos, presentes nas seis espécies (Ogura et al., 1976, 1977a, 1977 b; Prakash \& Garg, 1980; Ali et al., 1998); quinonas obtidas de Jacaranda caucana (Ogura et al., 1976, 1977a), Jacaranda copaia e Jacaranda mimosifolia (Sauvain et al., 1993); flavonóides isolados de Jacaranda decurrens (Blatt et al., 1998), Jacaranda acitufolia (Ferguson \& Lien, 1982), Jacaranda mimosifolia (Subramanian et al., 1973; Moharram \& Marzouk, 2007); ácidos graxos isolados de Jacaranda filicifolia e Jacaranda mimosifolia (Chisholm \& Hopkins, 1962; Ali et al., 1998) e acetosídeos e feniletanóides isolados de Jacaranda mimosifolia (Moharram \& Marzouk, 2007).

De acordo com Copp (2003), os metabólitos secundários considerados como a principal classe promissora de atividade antimicobacteriana são os terpenóides.

Segundo estudos realizados por Higuchi et al. (2008), os principais terpenóides isolados e que apresentaram atividade inibitória do crescimento do Micobacterium foram o lupeol, ácido ursólico e ácido oleanólico. Acido ursólico e o ácido oleanólico também foram isolados de algumas espécies de Jacaranda (Gachet \& Schüly, 2009). A presença dessa classe de metabólitos nos extratos metanólicos das cascas e das folhas de Jacaranda cuspidifolia 
sugerem a provável hipótese que essas substâncias sejam uma das responsáveis pela ação antimicobacteriana desses extratos.

A caracterização dos extratos metanólicos das cascas e das folhas de $J$. cuspidifolia foi realizada também por CLAE-FR (Figura 1A). A cromatografia líquida de alta eficiência (CLAE) é utilizada em trabalhos de fitoquímica para monitorar as etapas de isolamento, bem como, avaliar a pureza final das substâncias isoladas. Além disso, o perfil cromatográfico obtido por CLAE para tinturas e extratos vegetais, em condições padronizadas, denominadas "impressão digital", revela a constituição qualitativa e permite caracterizar o material analisado. Assim nos perfis cromatográficos dos extratos metanólicos das cascas e folhas de J. cuspidifolia observou-se a presença de substâncias de média polaridade, com predominância de picos em intervalo de 15 a 30 minutos, porém não foram observados picos com intensidades significativas após esse intervalo de tempo.

Os perfis dos extratos metanólicos das cascas e folhas foram semelhantes e mostraram a predominância de 7 picos, com tempos de retenção de 16,45 (1), 16,92 (2), 18,41 (3), 21,78 (4), 23,77 (5), $27,60(6), 30,51$ (7) min. Os espectros de ultravioleta obtidos on line para esses picos indicaram absorção em comprimento de onda de 275,$7 ; 273,3$ e 281,6 nm para os picos (1), (2) e (3), respectivamente. Já para os picos (4), (6) e (7) indicaram absorção em comprimento de onda similar e na faixa de 217,8 ; 250; 290 e 330,4 nm, e na faixa de 217,8; 250; 290 e $326,8 \mathrm{~nm}$ para o pico (5).

Estes comprimentos de onda são compatíveis com ariletanóides de cinamoil glicosídeos. O Vebascosídeo foi analisado como composto de referência nas mesmas condições dos extratos (Figura 1B). O cromatograma apresentou pico com tempo de retenção de 21,80 min e espectro no ultravioleta com comprimento de onda máximo de 217,8; 250; 290 e 331,6 nm, demonstrando assim que as principais substâncias presentes nos extratos metanólicos das cascas e folhas de Jacaranda cuspidifolia pertencem a essa classe.

Vários trabalhos têm demonstrado que o verbascosídeo e derivados estão distribuídos em muitas espécies da família Bignoniaceae e exibem várias atividades biológicas (Garcez et al., 2007; Santoro et al., 2008). A atividade antimicrobiana foi comprovada por Ávila et al. (1999) e Rigano et al. (2006). A presença desta classe de metabólitos nos extratos metanólicos das cascas e folhas de Jacaranda cuspidifolia também sugerem a provável hipótese que essas substâncias sejam uma das responsáveis pela ação antimicobacteriana desses extratos.

Os resultados obtidos através deste estudo corroboram com o uso popular de Jacaranda

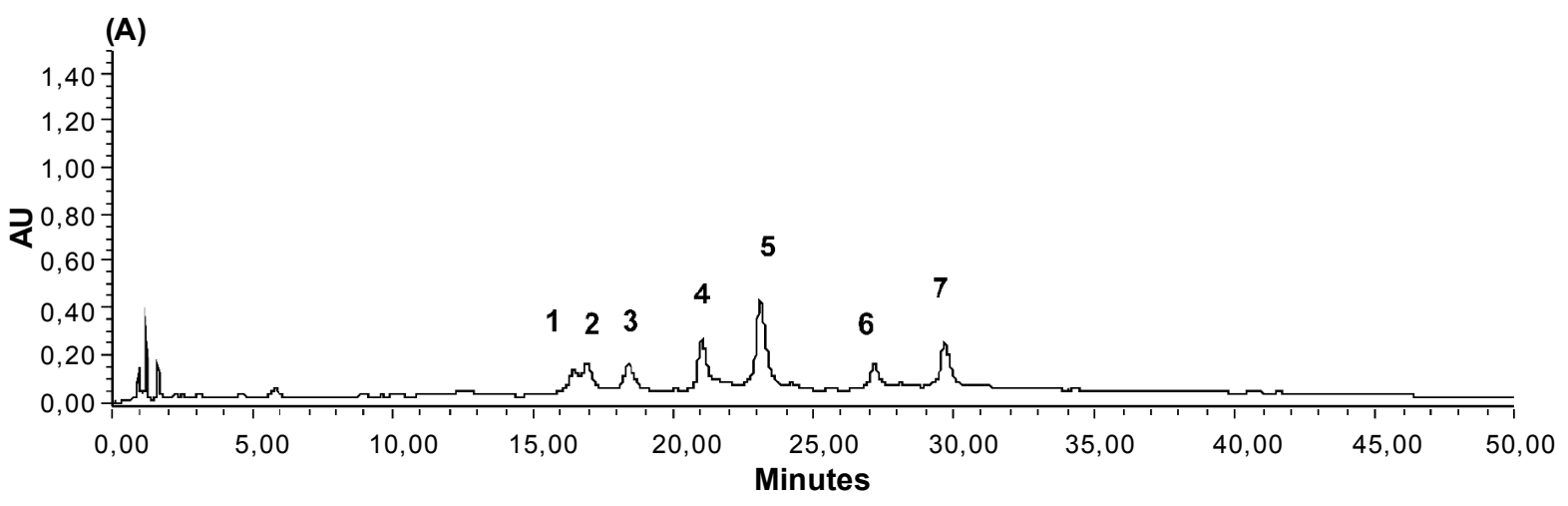

(B)

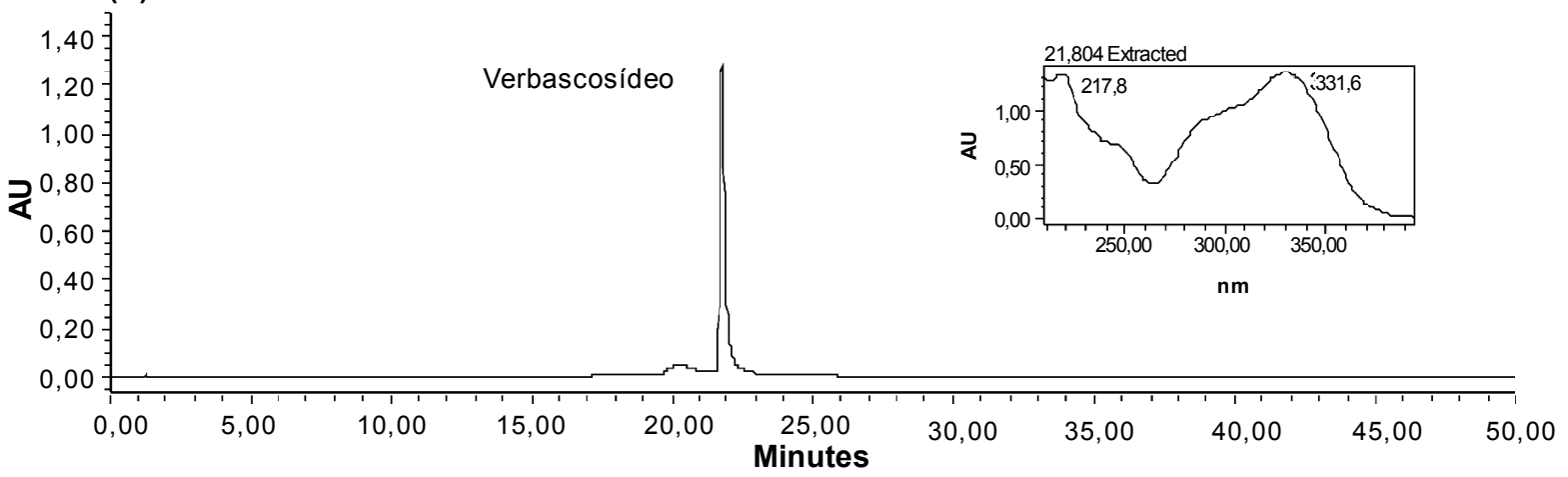

FIGURA 1. Perfil CLAE-FR do EMC (A) de Jacaranda cuspidifolia Mart. e do Verbascosídeo (B). Condições CLAE ver parte experimental. 
cuspidifolia Mart. no tratamento de infecções causadas por microrganismos e também demonstram a presença de substâncias da classes dos terpenóides e dos ariletanóides de cinamoil glicosídeos como as prováveis substâncias responsáveis pela ação antimicrobiana.

\section{AGRADECIMENTO}

Os autores agradecem a Fundação de Apoio ao Desenvolvimento de ensino, Ciência e Tecnologia do Estado de Mato Grosso do Sul (FUNDECT); Coordenação de Aperfeiçoamento de Pessoal de Nível Superior (CAPES); Fundação de Amparo à Pesquisa do Estado de Minas Gerais (FAPEMIG) e a Universidade Católica Dom Bosco (UCDB) pelo apoio financeiro.

\section{REFERÊNCIA}

ALI, R.M. et al. Antifungal activity of some Bignoniaceae found in Malaysia. Phytotherapy Research, v.12, n.5, p.331-4, 1998.

ALMEIDA, S.P. et al. Cerrado: espécies vegetais úteis. Planaltina: Embrapa, 1998. p.38-9.

ARANTES, V.P. et al. Plantas do Cerrado Brasileiro com atividade contra Mycobacterium fortuitum. Revista de Ciências Farmacêuticas Básica Aplicada, v.26, n.3, p.195-8, 2005.

ASRES, K. et al. Investigations on antymicobacterial activity of some Ethiopian medicinal plants. Phytoterapy Research, v.15, n.4, p.323-6, 2001.

AVILA, J.G. et al. Mode of action of Buddleja cordata verbascoside against Staphylococcus aureus. Journal of Ethnopharmacology, v.66, n.1, p.75-8, 1999.

BLATT, C.T.T. et al. Flavonoids of Bignoniaceae from the Cerrado and their possible taxonomic significance. Plant Systematics and Evolution, v.210, n.3-4, p.289-92, 1998. CANTRELL, C.L. et al. Antimycobacterial plant terpenoids. Planta Medica, v.67, p.1-10, 2001.

CHISHOLM, M.J.; HOPKINS, C.Y. Isolation and structure of a new conjugated triene fatty acid. Journal of Organic Chemistry, v.27, n.9, p.3137-9, 1962.

COPP, B.R. Antimycobacterial natural products. Natural Products Report, v.20, n.6, p.535-57, 2003.

DÍAZ, J.C.R. Synergic activity of flouoroquinolones and linezolid against Mycobacterium tuberculosis. International Journal of Antimicrobial Agents, v.21, n.5, p.354-6, 2003.

FERGUSON, N.M.; LIEN, E.J. A flavonol neohesperidoside from Jacaranda acutifolia. Journal of Natural Products, v.45, n.5, p.523-4, 1982.

FRANZBLAU, S.G. Rapid, low-technology MIC determination with clinical Mycobacterium tuberculosis isolates by using the microplate Alamar Blue assay. Journal Clinical Microbiology, v.36, p.362-6, 1998.

FRIS-MOLER, A. In vitro antimycobacterial and antilegionella activity of licochalcone $A$ from Chinese licorice roots. Planta Medica, v.68, p.416-9, 2002.

GACHET, M.S.; SCHÜLY, W. Jacaranda - An ethnopharmacological and phytochemical review. Journal of Ethnopharmacology, v.121, n.1, p.14-27, 2009. GARCEZ, F.R. et al. Novos constituintes químicas das cascas do caule de Tabebuia heptaphylla. Química Nova, v.30, n.8, p.1887-91, 2007.

GIBBONS, S. et al. Cryptolepine hydrochloride: a potent antimycobacterial alkaloid derived from Cryptolepis sanguinolenta. Phytoterapy Research, v.17, n.4, p.4346, 2003.

HIGUCHI, C.T. et al. Triterpenes and antitubercular activity of Byrsonima crassa. Química Nova, v.31, n.7, p.171921, 2008.

JANUÁRIO, A.H. et al. Antimycobacterial physalins from Physalis angulata L. (Solanaceae). Phytoterapy Research, v.16, n.5, p.445-8, 2002.

LORENZI, H. Árvores Brasileiras. 3.ed. Nova Odessa: Plantarum, 2000. 352p.

KANOKMEDHAKUL, $S$. et al. Antimycobacterial anthraquinone-chromanone compound and diketopiperazine alkaloid form the fungus Chaetomuim globosum KMITL-N0802. Planta Medica, v.68, p.834-6, 2002.

MAHRAN, G.H. et al. Phytochemical and antimicrobial study of Jacaranda mimosaefolia D. Don grown in Egypt. Herba Hungarica, v.30, p.98-104, 1991.

MCGAW, L.J. et al. Purified compounds and extracts from Euclea species with antymicobacterial activity against Mycobacterium bovis and fast-growing mycobacteria. Biological and Pharmaceutical Bulletin, v.31, p.1429-33, 2008.

MOHARRAM, F.A.; MARZOUK, M.A.S. Anovel phenylethanoid dimer and flavonoids from Jacaranda mimosaefolia. Zeitschrift für Naturforschung, v.62, p.1213-20, 2007.

MURILLO, F.I. et al. Antimycobacterial flavones from Haplopappus sonorensis. Fitoterapia, v.74, p.226-30, 2003. NEWTON, S.M. et al. The evaluation of forty-three plant species antimycobacterial activities; isolation of active constituents from Psoralea corylifolia and Sanguinaria canadensis. Journal of Ethnopharmacology, v.70, n.1, p.5767, 2002.

OKUNADE, A.L. et al. Natural antimycobacterial metabolites: current status. Phytochemistry, v.65, n.8, p.1017-32, 2004. OGURA, M.; CORDELL, G.A.; FARNSWORTH, N.R. Potential anticancer agents. IV. Constituents of Jacaranda caucana Pittier (Bignoniaceae). Lloydia, v.40, p.157-68,1977a. OGURA, M.; CORDELL, G.A.; FARNSWORTH, N.R. Jacoumaric acid, a new triterpene ester from Jacaranda caucana. Phytochemistry, v.16, n.2, p.286-7, 1977 b.

ORGANIZAÇÃO MUNDIAL DA SAÚDE. Global tuberculosis control: epidemiology, strategy and financing. Geneva: OMS, 2009. 28p.

PARKER, T. Trees of Guatemala. Austin: The Tress Press, 2008. p.86-7/90-1.

PAVAN, F.R. et al. In vitro anti-Mycobacterium tuberculosis activity of some Braziliam "Cerrado" plants. Brazilian Journal of Pharmacognosy, v.19, n.1, p.204-6, 2009.

PRAKASH, L.; GARG, G. Chemical examination of the root barks of Jacaranda mimosaefolia D. Don. and Tabebuia pentaphylla (Linn) Hemsl. Pharmazie, v.35, n.10, p.649, 1980.

POTT, A.; POTT, V.J. Plantas do Pantanal. Corumbá: Empresa Brasileira de Pesquisa Agropecuária do Pantanal, 1994. 320p. 
RANDO, D.G. et al. Potential tuberculostatic agents, topliss aplication on benzoic acid. Bioorganic \& Medicinal Chemistry, v.10, n.3, p.557-60, 2002.

RIGANO, D. et al. Antibacterial activity of flavonoids and phenylpropanoids from Marrubium globosum ssp. libanoticum. Phytoterapy Reasearch, v.21, n.5, p.395-7, 2006. RODRÍGUEZ, J.C. et al. In vitro activity of four fluoroquinolones against Mycobacterium tuberculosis. International Journal of Antimicrobial Agents, v.17, p.229-31, 2001.

SANKARA-SUBRAMANIAN, S.; NAGARAJAN, S.; SULOCHANA, N. Flavonoids of eight Bignoniaceous plants. Phytochemistry, v.11, n.2, p.1499, 1972.

SUBRAMANIAN, S.S.; NAGARAJAN, S.; SULOCHANA, N. Hydroquinone from the leaves of Jacaranda mimosaefolia. Phytochemistry, v.12, n.4, p.220-1, 1973. SANTORO, A. et al. Verminoside and verbascoside induced genotoxicity on human lymphocytes: Involvement of PARP-1 and p53 proteins. Toxicology Letters, v.178, n.2, p.71-6, 2008.

SAUVAIN, M. et al. In vitro and in vivo leishmanicidal activities of natural and synthetic quinoids. Phytotherapy
Research, v.7, p.167-71,1993.

SCALON, S.P.Q. et al. Armazenamento e tratamentos prégerminativos em sementes de jacarandá (Jacaranda cuspidifolia Mart.). Revista Árvore, v.30, n.2, p.179-85, 2006. SCHINKOVITZ, A. et al. Ostruthin: An antimycobacterial coumarin from the roots of Peucedanum osthruthium. Planta Medica, v.69, p.369-71, 2003.

SEEPHONAKI, P. et al. Evaluation of antimycobacterial, antiplasmodial and cytotoxic activities of preussomerins isolated from the lichenicolous fungus Microsphaeropsis sp. BCC 3050. Planta Medica, v.68, p.45-8, 2002.

$\mathrm{SCHI}$, Y.P. et al. New terpenoid constituents from Eunicea pinta. Journal Natural of Products, v.65, n.9, p.1232-41, 2002.

SOUZA, M.V.N.; VASCONCELOS, T.R.A. Fármacos no combate à tuberculose: passado, presente e futuro. Química Nova, v.28, n.4, p.678-82, 2005.

SUNTHITIKAINSAKUL, A. et al. Coumarins and carbazoles from Clausena excavata exhibited antimycobacterial and antifungal activities. Planta Medica, v.69, p.155-7, 2003. WAGNER, H. et al. Plant drug analysis. Berlin: SpringerVerlag. 1984. 320p. 